Revista de Psicología Vol. 33 (1), 2015 (ISSN 0254-9247)

\title{
Empoderamiento psicológico: un modelo sistémico con componentes individuales y comunitarios
}

\author{
Ana Lilia Banda Castro ${ }^{1}$ y Miguel Arturo Morales Zamorano ${ }^{2}$ \\ Universidad de Sonora - México
}

Se busca confirmar que la participación social puede influir en el empoderamiento como uno de sus cuatro componentes. La hipótesis propone la interacción sistémica de dos componentes del empoderamiento: intrapersonal y comportamental. Los participantes fueron 113 habitantes urbanos a quienes se les aplicó una encuesta. Los resultados evidencian que el modelamiento estructural identificó un componente intrapersonal del empoderamiento integrado por empoderamiento positivo, empoderamiento negativo y control sociopolítico. Este componente se muestra afectado por el componente de comportamiento conformado por la organización social, las acciones comunitarias y la toma de decisiones. Se acepta la hipótesis y se proporciona sustento empírico a la teoría.

Palabras clave: agencia, componente intrapersonal, componente de comportamiento, empoderamiento psicológico, participación social

Psychological Empowerment: A systemic model with individual and community components

This study aimed to confirm that social participation may influence empowerment as one of the four components explained by the theory. The hypothesis proposes systemic interaction of two components of empowerment: intrapersonal and behavioral. 113 urban residents participated in the study. The hypotheses were tested through the use of structural modeling. An intrapersonal component of empowerment, composed of positive empowerment, negative empowerment and socio-political control was identified. This component was affected by the behavioral component that involves social organization, community action and decision making. The hypothesis is accepted and the study provides empirical support to the theory. Keywords: Agency, intrapersonal component, behavioral component, psychological empowerment, social participation

1 Doctora en Ciencias Sociales por la Universidad Autónoma de Sinaloa, profesora investigadora titular. Dirección postal: Cerrada San Fernando \# 14 C.P. 83249, Col. Capistrano, Hermosillo Sonora, México. Contacto: albanda@psicom.uson.mx

2 Doctor en Ciencias de la Administración por el Instituto Politécnico Nacional, profesorinvestigador titular. Dirección postal: Avenida Diez No. 89, entre calles Tres y Perimetral, C.P. 83140, Col. Bugambilias, Hermosillo, Sonora. Contacto: moralesz@sociales.uson.mx 

El término empoderamiento se vincula con otros términos como el fortalecimiento, la potenciación, el reempoderamiento o el apoderamiento, y con él se introduce la noción de poder no solo como fenómeno social, sino también como variable psicológica. A su vez, el empoderamiento no solo es un concepto operativo, sino que también representa un valor que es la base de la transformación cultural (Sánchez Vidal, 2013).

Según Maton (2008), el escenario comunitario que favorezca el empoderamiento debe reunir ciertas condiciones, entre las que se encuentran: el sistema de creencias en base al grupo, las actividades centrales que desarrolla la comunidad, el ambiente que favorece las relaciones, la estructura de oportunidad para el ejercicio de roles, liderazgo, cambio, así como el funcionamiento del escenario comunitario. Este último autor señala que existen cuatro áreas de dominio para el empoderamiento. La primera área se refiere al empoderamiento que llevan a cabo los adultos y que les permite superar dificultades individuales. La segunda área de dominio tiene que ver con el empoderamiento que los jóvenes son capaces de promover ante circunstancias adversas de desarrollo, realización y ejecución. La tercera área de dominio hace referencia al empoderamiento de los ciudadanos en comunidades empobrecidas, tomando acciones para mejorar las comunidades en las que viven. Y la cuarta área de dominio del empoderamiento se enfoca al empoderamiento histórico de ciudadanos oprimidos que resisten y desafían a la sociedad, la cultura y las instituciones (Maton, 2008).

El empoderamiento es considerado el proceso mediante el cual las personas, organizaciones y comunidades logran control sobre sus asuntos. Es así que las personas, organizaciones o grupos más impotentes y marginados se dan cuenta de la función del poder dentro del contexto de sus vidas, y desarrollan habilidades y capacidades para mejorar el control de las mismas. Este control puede ser ejercido sin perjudicar 
los derechos de los demás. Asimismo, involucra cambios en la identidad y en la autopercepción de las personas. Generalmente cuando un individuo o grupo se empoderan, se apoya en la comunidad para la transformación de su entorno según sus necesidades y aspiraciones, así como para sí mismos (Montero, 2009a).

El empoderamiento es un proceso y puede tener el carácter de un resultado. Ambas condiciones conferidas al empoderamiento se generan en las interacciones entre las personas, en donde el resultado es el inicio de una nueva fase del proceso de empoderamiento y, por consiguiente, resulta muy difícil establecer una clara diferenciación del empoderamiento como proceso o como resultado. Sin embargo, es precisamente este carácter dinámico el que hace que entornos en donde prevalecen individuos empoderados, se conviertan en entornos empoderadores, lo cual es atribuible a la influencia del entorno. En este caso, el empoderamiento psicológico ha sido teorizado de acuerdo con una perspectiva ecológica de desarrollo que ubica el análisis del empoderamiento desde un punto de vista psicológico, organizacional y comunitario, que se verá afectado por el contexto ecológico o cultural en que se manifiesta. Por ello, los entornos son los que matizarán la naturaleza del mismo. Finalmente, el empoderamiento psicológico variará de acuerdo con el tiempo, su condición no es estática, por lo que puede mermarse o incrementarse (Christens, 2012a; Frain, Tschopp \& Bishop, 2009).

El empoderamiento, además de abarcar las autopercepciones de competencia, incluye el compromiso activo dentro de la comunidad, la comprensión de su entorno sociopolítico, el aprendizaje sobre los agentes de control y la acción para influenciar a esos agentes. De acuerdo con una red nomológica de empoderamiento, en un nivel individual de análisis se encuentra el empoderamiento psicológico (Maton, 2008; Montero, 2009a), el cual es definido como un sentido de competencia personal, un deseo o disponibilidad por tomar acciones en dominios públicos que se correlaciona negativamente con la alineación y positivamente con el liderazgo. El empoderamiento psicológico se compone por dimensiones como son la personalidad, los aspectos cognitivos y los aspectos motivacionales del control personal, la autoestima, la toma 
de decisiones, y la autoeficacia (Bennett \& Chapman, 2010; Montero, 2009b; Wang, Chen \& Chen, 2011); así como por percepciones de control y autoeficacia en la esfera sociopolítica (Peterson et al., 2006).

La participación, el control y la conciencia crítica son los resultados producidos por el empoderamiento psicológico según Peterson y colaboradores (2006), lo que se testifica colectivamente con el ejercicio de cada individuo empoderado. Se han contemplado cuatro componentes del empoderamiento psicológico: el emotivo o intrapersonal, el cognitivo o interaccional, el de comportamiento y el interpersonal o relacional (Christens, 2012b). El componente intrapersonal se refiere a la capacidad percibida por el ser humano para influir en los sistemas sociales y políticos. Esta autopercepción incluye un dominio específico del control percibido, el autoconcepto, la autoeficacia, autoestima, la motivación para ejercer control y los sentimientos de competencia que alentarán a las personas a actuar y participar (Christens, Speer \& Peterson, 2011b; Speer, Peterson, Armstead \& Allen, 2013). En el plano individual el empoderamiento incluye además comprensión del ambiente sociopolítico (Peterson et al., 2006).

Pick y colaboradores (2007), en un estudio realizado con población mexicana urbana y rural, han identificado dos factores: agencia personal y empoderamiento. Los mecanismos asociados a estos fueron la autoeficacia, la autodeterminación, la autonomía, el pensamiento independiente, el miedo al éxito y la identificación de necesidades de cambio. Estos autores diferencian la agencia personal del empoderamiento, al seńalar que la primera repercute internamente en el individuo y el segundo impacta el exterior. La literatura científica no ha permitido corroborar tal diferenciación y por el contrario se ha encontrado que el componente intrapersonal del empoderamiento se integra por variables que impactan internamente o externamente al individuo (Peterson et al., 2006; Speer et al., 2013). Para Tejada (2005) la agencia humana tiene un carácter instrumental como también le ha sido conferido al empoderamiento, ya que de acuerdo con este autor la agencia humana permite al individuo seleccionar, estructurar y crear ambientes que optimicen el aprendizaje y que posibiliten encontrar 
alternativas de transformación tanto en el entorno como entre los individuos mismos.

El empoderamiento incrementa la satisfacción y confianza de los individuos, ya que se pueden reconocer los efectos o resultados que ocasionan las propias acciones, aumenta la creatividad por que se reconoce la autonomía con que se desenvuelve el ser humano y se reduce la resistencia al cambio ya que las personas se encuentran involucradas (Chiavola, Cendrós Parra \& Sánchez, 2008). Sin el componente intrapersonal de empoderamiento, el individuo se puede ver expuesto a experimentar sentimientos de apatía, impotencia, carencia de control e indefensión aprendida (Speer et al., 2013). Un estudio longitudinal (Christens, Peterson \& Speer, 2011a) ha permitido encontrar que los procesos de empoderamiento intrapersonal dan por resultado que los individuos se socialicen mediante su participación en entornos comunitarios empoderados.

El componente de comportamiento se refiere a acciones específicas que se dirigen a satisfacer necesidades específicas del ambiente y que ejercen influencia en los entornos sociales y políticos, mediante la participación en organizaciones y actividades de la comunidad (Bennett $\&$ Chapman, 2010). Dependiendo del contexto, los comportamientos de empoderamiento pueden implicar desde ajustes individuales hasta condiciones adversas que no son maleables para el cambio, para el trabajo con otros dentro de una organización de voluntariado, o para alterar la distribución de los recursos comunitarios.

Las acciones comunitarias de la participación social pueden ser atribuidas al empoderamiento psicológico (Banda, Valenzuela, González \& Morales, 2010; Banda, Morales, Flores, Del Castillo \& Quintero Mármol, 2012; Ríos \& Moreno, 2009). Otra dimensión de la participación social que se vincula con el componente de comportamiento la constituye la organización social, la cual se caracteriza por la participación de los individuos en organizaciones que contribuyen al mejoramiento de la sociedad, tales como: asociaciones vecinales, grupos políticos, grupos de autoayuda, grupos religiosos u organizaciones de servicios por citar algunos ejemplos (Banda et al., 2010; Banda 
et al., 2012; Ríos \& Moreno, 2009; Vidoni, Mascherini \& Manca, 2009).

Para los ciudadanos, el empoderamiento de las comunidades se realiza mediante la participación social que conduce a la toma de decisiones. A su vez, es posible que la ciudadanía se oriente hacia ciertas acciones de recopilación y de análisis de información que la conducen a tomar riesgos o beneficios que las mismas acciones conlleven. Sin tener la posibilidad de delimitar el orden secuencial entre la toma de decisiones y la participación, social es innegable que ambos procesos logran persuadir a las autoridades gubernamentales y posibilitan que los ciudadanos desempeñen un papel activo (Angulo, 2010; Christens, 2012b; Ríos \& Moreno, 2009).

La teoría de empoderamiento reconoce que el ajuste entre las características individuales (por ejemplo la raza o los ingresos) y los procesos potencialmente empoderadores pueden tener efectos vitales para el desarrollo de los resultados del empoderamiento. En un primer hallazgo se encontró que la participación social contribuye con el empoderamiento intrapersonal (empoderamiento emocional) en grupos con diversidad de ingresos económicos (Speer et al., 2013). Mientras que en un segundo hallazgo se observó que los grupos de bajos ingresos muestran interacción entre la participación social y el componente de empoderamiento cognitivo. Estos dos hallazgos son una especificación de los diferentes resultados del empoderamiento, ya que previamente se había señalado que la participación social antecede al empoderamiento (Speer et al., 2013).

Speer y colaboradores (2013) señalan que existen diferencias en el mecanismo de participación que se desarrollan de acuerdo con el género, de tal forma que personas con puntaje alto en empoderamiento del sexo masculino son más tendientes a participar en las funciones o roles que son representativos para los demás. Asimismo, los puntajes de empoderamiento altos siendo del sexo femenino se asocian con el compromiso con otros, en los procesos de toma de decisiones organizacional.

Conforme a todo lo expuesto, la presente investigación tiene como objetivo identificar dos componentes de la teoría de empoderamiento 
mediante la aplicación de una encuesta de opción múltiple, así como especificar la influencia que prevalece entre el componente intrapersonal y el componente de comportamiento.

\section{Método}

\section{Participantes}

La muestra estuvo formada por 113 habitantes del sur de la ciudad de Hermosillo, Sonora, México, seleccionados por colonias de acuerdo con el Instituto Nacional de Geografía e Informática INEGI. Ello debido a que estas colonias contenían la mayor cantidad de habitantes con ingresos mensuales menores de 1600 pesos (123 dólares aproximadamente). La selección de la muestra con reducidos ingresos se fundamenta en los planteamientos de Montero (2009a, 2009b), que señala que las personas que cuentan con este rango de ingresos son los más comúnmente aquejados por procesos sociales desfavorables como la carencia empoderamiento, la falta de participación social y/o el limitado desarrollo social. Los criterios de inclusión se circunscribieron a elegir participantes de sexo indistinto, mayores de 18 ańos de edad, habitantes de las colonias o barrios visitados y que accedieran a cooperar en el estudio. Los criterios de exclusión fueron que las personas invitadas a participar no vivieran en la colonia o barrio, fueran menores de edad o que no accedieran a participar. Se acudió a las colonias previamente definidas realizando un muestreo no probabilístico por conveniencia. Las edades oscilaron entre 18 y 85 ańos, y la media de edad de los participantes fue de 38 años $(D E=14.55)$, con escolaridad promedio de segundo año de secundaria. La muestra se integró por 57 mujeres (50.4\%) y 56 hombres (49.5\%).

\section{Medidas e instrumentos de medición}

Para medir el componente de comportamiento se empleó la escala de participación social con 59 reactivos, los cuales registran dimensiones de participación social relacionadas con: las acciones comunitarias, la toma de decisiones y la organización social (Banda et al., 2012). 
Esta escala previamente ha sido aplicada en otra muestra en la misma ciudad para otro estudio. Las propiedades psicométricas han sido analizadas con 400 casos, obteniéndose alfas de Cronbach por dimensión que oscilaron entre .70 y .98. La validez de contenido de la escala de participación social fue obtenida mediante la presentación de los reactivos a un grupo de cinco expertos. Adicionalmente, se realizó análisis factorial de la escala, a partir del cual se identificaron las dimensiones previamente señaladas, por lo que el instrumento cuenta con validez de constructo para una muestra mexicana.

Para medir el componente intrapersonal vinculado a la dimensión de control sociopolítico, se empleó una escala con el mismo nombre integrada por 12 reactivos, que evaluaron la competencia en el liderazgo y las políticas de control, a las cuales les correspondieron los valores de alfa de Cronbach de .78 y .81 respectivamente obtenidos por sus autores (Peterson et al., 2006).

Para registrar el componente intrapersonal con las dimensiones de agencia o empoderamiento positivo, y agencia o empoderamiento negativo, se emplearon 17 reactivos de la escala que mide agencia personal y el empoderamiento (ESAGE), los cuales se encuentran asociados a variables como autodeterminación, autoeficacia, control del comportamiento (Pick et al., 2007). Esta escala ha sido aplicada por sus autores obteniendo alfas de Cronbach con valor de .71. Adicionalmente, los autores realizaron análisis factorial, identificando los dos factores previamente hipotetizados en una muestra mexicana.

\section{Procedimiento}

Las encuestas fueron realizadas durante diversas sesiones por psicólogos entrenados previamente en la administración de las mismas. Con la consigna de distribuirse por las calles de las colonias previamente seleccionadas, los psicólogos solicitaron consentimiento informado para encuestar a los habitantes con residencia en dichas colonias. Las sesiones duraron aproximadamente una hora y solo fueron realizadas cuando los participantes accedieron a hacerlo y fueron cubiertos los criterios de inclusión. 


\section{Análisis de datos}

Se verificó la confiabilidad (consistencia interna) de las dimensiones que integraron cada escala obteniendo las alfas de Cronbach en el paquete estadístico System of Statistical Analysis [SAS] (versión 9.1).

Los datos recabados fueron contrastados con el modelo teórico mediante el paquete estadístico Structural Equations [EQS] (versión 6). Se realizó la técnica de análisis factorial confirmatorio AFC de los modelos de ecuaciones estructurales ya que permite poner a prueba las hipótesis antes referidas que se vinculan al modelo (Ferrando \& Anguiano, 2010). El modelo va de acuerdo con el principio de parsimonia ya que el número de factores es más pequeño que el número de variables (Ferrando \& Anguiano, 2010; Herrero, 2010). Para evaluar el modelo de ecuaciones estructurales se obtuvo el estadístico chi cuadrado $\left(c^{2}\right)$ esperando que dichos valores fueran bajos y con niveles de significación mayores que .01 (Wothke, 2010), en virtud de que se buscaba coincidencia entre el modelo teórico con el observado. Como opción para corregir la tendencia del estadístico chi-cuadrado para rechazar cualquier modelo especificado con una muestra suficientemente grande (entre 100 a 200 participantes), se obtuvo el error de aproximación cuadrático medio RMSEA, cuyos valores aceptables oscilaran entre .05 y .08 (Ruíz, Pardo \& San Martín, 2010). Adicionalmente, se consideraron los siguientes índices: Bentler-Bonnet de ajuste normado BBN, Bentler-Bonett de ajuste no normado BBNN y el índice de ajuste comparativo CFI.

\section{Resultados}

La confiabilidad de los reactivos por consistencia interna calculada mediante el coeficiente alpha de Cronbach arroja los siguientes valores para la dimensión del componente de comportamiento o participación social: acciones comunitarias $(\alpha=.57)$, toma de decisiones $(\alpha=.83)$, organización social $(\alpha=.66)$. Para las dimensiones del componente intrapersonal los coeficientes obtenidos fueron empoderamiento 
positivo $(\alpha=.58)$, empoderamiento negativo $(\alpha=.52)$ y control sociopolítico $(\alpha=.83)$.

Mediante la estimación por máxima verosimilitud que utiliza el modelamiento de ecuaciones estructurales (Closas, Arriola, Kuc, Amarilla \& Jovanovich, 2013; Wothke, 2010), se registró el indicador global chi cuadrado $c^{2}$ para el modelo propuesto que fue de $14.60(g l=8$, $p>.06)$. Los valores de los estadísticos fueron: RMSEA (.08), índice de BBN (.92), el índice de BBNN (.92) y el índice de ajuste comparativo CFI (.96). Se empleó el análisis factorial confirmatorio que permitió contrastar el modelo teórico en donde las dimensiones de cada escala fueron transformadas a factores mediante el análisis factorial confirmatorio (Closas et al., 2013; Ruíz et al., 2010).

El modelo constó de dos factores el componente intrapersonal y el de comportamiento lo cual fue contrastado mediante un análisis factorial confirmatorio. Dicho AFC utilizó las dimensiones latentes provenientes de estos dos factores obteniendo los respectivos pesos factoriales significativos como se muestra en la Tabla 1 y en la Figura 1.

En la Figura 1, según los indicadores BBN, BBNN, CFI, y RMSEA, el modelo propuesto presenta un adecuado ajuste a los datos (Herrero, 2010; Ruíz et al., 2010) los valores estadísticos se encuentran dentro de los rangos aceptables y sugieren que la estructura factorial puede representar los constructos propuestos. Los pesos factoriales de cada una de las dimensiones de cada factor medido alcanzaron valores superiores a .46 , por lo que se consideran moderadamente altos (Herrero, 2010).

\section{Discusión}

Los resultados recabados mediante este estudio han permitido medir dos componentes de empoderamiento psicológico: el componente intrapersonal y el componente de comportamiento (ver Tabla 1). 


\section{Tabla 1}

Matriz de pesos factoriales de las dimensiones por factor

\begin{tabular}{lc}
\hline Componente & Peso factorial \\
\hline Intrapersonal & \\
empoderamiento positivo & .63 \\
empoderamiento negativo & -.54 \\
Control sociopolítico & .46 \\
\hline Comportamiento & \\
Toma de decisiones & .68 \\
Organización social & .77 \\
Acciones comunitarias & .91 \\
\hline
\end{tabular}

Se han identificado las siguientes tres variables que integran el componente de comportamiento: organización social, referido por estudios previos (Banda et al., 2010; Banda et al., 2012; Ríos \& Moreno, 2009; Vidoni et al., 2009); acciones comunitarias y toma de decisiones, lo cual se sustenta con la investigación previa de Ríos y Moreno (2009). Con anterioridad, los componentes identificados y las variables que los integran han sido también identificados por varios estudios (Christens et al., 2011a, Christens et al., 2011b, Peterson et al., 2006; Speer et al., 2013).

El componente intrapersonal se conformó por el control sociopolítico, lo cual fue referido previamente por otras investigaciones (Christens et al., 2011a, Christens et al., 2011b, Peterson et al., 2006; y Speer et al., 2013). También se vinculan con el componente intrapersonal o emotivo variables como la agencia o empoderamiento positivo y la agencia o empoderamiento negativo, las cuales se encuentran asociadas a mecanismos de autodeterminación, autoeficacia, control del comportamiento, autoestima, autoconcepto (Bennett \& Chapman, 2010; Montero, 2009b; Wang et al., 2011; Pick et al., 2007). De acuerdo con esto último el componente intrapersonal o emotivo en este estudio, queda más específicamente medido ya que se le vincula con competencias y capacidades autopercibidas, en virtud de que usualmente este 
componente ha sido identificado mediante el registro del control sociopolítico únicamente (Christens et al., 2011a; Christens et al., 2011b; Peterson et al., 2006; Speer et al., 2013). Esta especificación de las competencias permite reafirmar que el empoderamiento, tal como han señalado Chiavola y colaboradores (2008) y por Christens, Peterson y Speer (2011a), incrementa la satisfacción, la confianza, la creatividad, la participación social y, a través del involucramiento de los individuos, reduce la resistencia al cambio, los sentimientos de apatía, la impotencia y la carencia de control (Speer et al., 2013).

Por consiguiente, en este estudio se realzan las características del empoderamiento que se compone de las competencias de autodeterminación, autoeficacia y autonomía, entre otras. Cabe señalar que el instrumento empleado para medir empoderamiento en el presente estudio aborda de manera genérica su análisis, motivo por el cual son necesarios continuar con estudios con poblaciones latinas aleatorizadas en donde se puedan investigar los componentes cognitivo o interaccional y el interpersonal o relacional (Christens, 2012b; Peterson et al., 2006), en adición al estudio del componente emotivo o intrapersonal y el de comportamiento. Ello a su vez proporcionará soporte empírico a la teoría y en especial al componente relacional que recientemente ha planteado Christens (2012b) (ver Figura 1).

En este estudio la participación social es contemplada teóricamente como un componente del empoderamiento psicológico, con el modelo empírico obtenido se registra que el componente de comportamiento se encuentra afectando el componente intrapersonal, esto coincide con lo reportado previamente por Christens (2012a) y por Speer y colaboradores (2013). Adicionalmente Christens (2012a) señala que el desarrollo participativo es visto como un promotor del empoderamiento psicológico. Esto significa que el contexto ecológico, cultural y los aspectos externos al individuo afectan el empoderamiento psicológico y, por consiguiente, las comunidades y las instituciones gubernamentales pueden promover o propiciar situaciones que se conviertan en ambientes empoderadores (Christens, 2012a; Frain et al., 2009; Montero, 2009a). Lo anterior sugiere a los autores de este trabajo 
que la práctica de comportamientos empoderados de inicio, pueden ser el ejemplo para transmitir nociones de empoderamiento psicológico con un carácter de resultado el cual podría posiblemente transformarse en el proceso dinámico ideal que debe caracterizar a todo individuo con potencial desarrollo humano (Montero, 2009a).

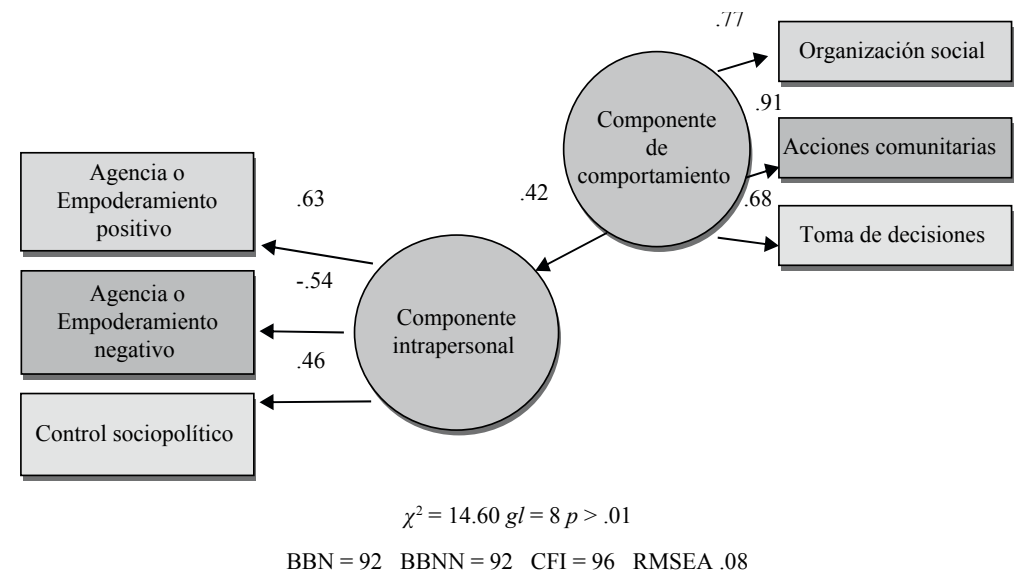

Figura 1. Modelo de empoderamiento psicológico

Los hallazgos de diversos autores, adicionados con los de este estudio, brindan soporte empírico a la teoría de empoderamiento y una aproximación a la generalización del conocimiento, en particular por haberse realizado en una población latina. La presente teoría brinda conocimiento sobre el empoderamiento, constructo integrado y afectado por diversos constructos de mayor o igual complejidad. En el presente estudio se permite analizar los constructos relacionados con el empoderamiento e identificar las relaciones entre los mismos, posibilitando el acercamiento a la explicación de fenómenos sociales como el empoderamiento, la participación social, el capital social y el desarrollo humano, que necesitan ser estudiados en futuras investigaciones para acercarnos a la comprensión del empoderamiento psicológico.

Las limitaciones de este estudio se concentran en que únicamente se planeó medir dos componentes del modelo teórico de Christens, 
Peterson y Speer (2011b), Peterson y colaboradores (2006), y Speer, Peterson, Armstead y Allen (2013). Asimismo, el tamaño de la muestra es pequeño y dirigido a personas con ingresos reducidos, por lo que la generalización de resultados debe ser contemplada con reservas. Por su parte, la escala ESAGE ha sido validada para la población mexicana, al igual que la escala de participación social. En el caso de la escala de control sociopolítico la validación no ha sido para la población estudiada, sin embargo todos los instrumentos fueron piloteados previamente y algunos de sus reactivos son empleados como indicadores del modelo empleado.

\section{Conclusiones}

A partir de los resultados obtenidos se concluye que es factible medir dos de los componentes del empoderamiento psicológico integrando mayor número de variables. A su vez, se ha identificado que el componente de comportamiento que tiene que ver con acciones específicas que se toman para ejercer influencia en el entorno, lo hace en el componente intrapersonal con lo que se brinda apoyo a la perspectiva ecológica y sistémica en que se ubica la teoría de empoderamiento. En esta investigación la participación social es considerada un componente del empoderamiento psicológico directamente relacionado con la teoría. El empoderamiento psicológico es un indicador de que los individuos están tomando acciones colectivas como componente comportamental, y este a su vez influye el componente intrapersonal para generar un cambio social o político. La consolidación o incremento del empoderamiento psicológico representa construir procesos de resiliencia que permitan a los individuos afrontar la adversidad, adaptarse a las nuevas situaciones u obstáculos y rehacerse, con lo cual se promueven los comportamientos positivos y saludables. Como una aproximación al establecimiento de políticas públicas, enfocarse en el empoderamiento psicológico, también equivale a resaltar los derechos ciudadanos y las necesidades de los individuos. 


\section{Referencias}

Angulo, S. (2010). Posibilidades y limitaciones para la participación social desde la perspectiva de distintos actores locales. Revista Reflexiones, 89(1), 67-75.

Banda, A. L., Morales, M. A., Flores, R., Del Castillo, D. M. \& Quintero-Mármol, A. (2012). La participación social mediadora en la calidad de vida de una población urbana. En R. Díaz-Loving, S. Rivera \& I. Reyes (Eds.), La Psicología Social en México (pp. 703708). México: Universidad Autónoma de Nuevo León, AMEPSO. Banda, A. L., Valenzuela, G. A., González, A. V. \& Morales, M. A. (2010). Factores individuales, sociales y políticos de participación ciudadana. En D. C. Gutiérrez, B. A. Valenzuela, J. Piovani \& M. Guillén (Eds.), Memoria del II Encuentro Latinoamericano de Metodología de las Ciencias Sociales (pp. 1-17). México: Universidad de Sonora.

Bennett, L. \& Chapman, A. R. (2010). The process of empowerment. American Psychologist, 65(7), 646-659.

Chiavola, C. Cendrós Parra, P. \& Sánchez, D. (2008). El empoderamiento desde una perspectiva del sistema educativo. Omnia, 14(3), 130-143.

Christens, B. D. (2012a). Targeting empowerment in community development: a community psychology approach to enhancing local power and well-being. Community Development Journal, 47(4), 538-554.

Christens, B. D. (2012b). Toward relational empowerment. American Journal of Community Psychology, 50(1-2), 114-128.

Christens, B. D., Peterson, N. A. \& Speer, P. W. (2011a). Community participation and psychological empowerment: testing reciprocal causality a cross-lagged panel design and latent constructs. Health Education \& Behavior, 38(4), 339-347.

Christens, B. D., Peterson, N. A. \& Speer, P. W. (2011b). Social class as moderator of the relationship between (dis) empowering 
processes and psychological empowerment. Journal of Community Psychology, 39(2), 170-182.

Closas, A. H., Arriola, E. A., Kuc, C. I., Amarilla, M. R. \& Jovanovich, E. C. (2013). Análisis multivariante, conceptos y aplicaciones en psicología educativa y psicometría. Enfoques $X X V(1)$, 65-92. Ferrando, P. J. \& Anguiano, C. (2010). El análisis factorial como técnica de investigación en psicología. Papeles del Psicólogo, 31(1), 18-33. Frain, M. P., Tschopp, M. K. \& Bishop, M. (2009). Empowerment variables as predictors of outcomes in rehabilitation. Journal of Rehabilitation, 75(1), 27-35.

Herrero, J. (2010). El análisis factorial confirmatorio en el estudio de la estructura y estabilidad de los instrumentos de evaluación: un ejemplo con el cuestionario de autoestima CA-14. Psychosocial Intervention, 19(3), 289-300.

Maton, K. I. (2008). Empowering community settings: agents of individual development, community betterment and positive social change. American Journal of Community Psychology, 41(12), 4-21.

Montero, M. (2009a). El fortalecimiento en la comunidad, sus dificultades y alcances. Universitas Psychologica, 8(3), 615-626.

Montero, M. (2009b). Community action and research as citizenship construction. American Journal of Community Psychology, 43(12), 149-161.

Peterson, N. A., Lowe, J. B., Hughey, J., Teid, R. J., Zimmerman, M. A. \& Speer, P. W. (2006). Measuring the intrapersonal component of psychological empowerment: confirmatory analysis of the sociopolitical control scale. American Journal of Community Psychology, 38(3-4), 287-297.

Pick, S., Sirkin, J., Ortega, I., Osorio, P., Martínez, R., Xocolotzin, U. \& Givaudan, M. (2007). Escala para medir agencia personal y empoderamiento. Revista Interamericana de Psicología, 41(3), 295-304.

Ríos, M. L. \& Moreno, M. P. (2009). Influencia de la participación comunitaria y la identidad con el lugar en la satisfacción vital en inmigrantes. Escritos de Psicología, 3(2), 8-16. 
Ruíz, M. A., Pardo, A. \& San Martín, R. (2010). Modelos de ecuaciones estructurales. Papeles del Psicólogo, 31(1), 34-45.

Sánchez Vidal, A. (2013). ¿Es posible el empoderamiento en tiempos de crisis? Repensando el desarrollo humano en el nuevo siglo. Universitas Psychologica, 12(1), 285-300.

Speer, P., Peterson, N., Armstead, T. \& Allen, C. (2013). The influence of participation, gender and organizational sense of community on Psychological Empowerment: The moderating effects of income. American Journal of Community Psychology, 51(1/2), $103-113$.

Tejada, A. (2005). Agenciación en la teoría cognitivo social: definición y posibilidades de aplicación. Pensamiento psicológico, 1(5), 117 123.

Vidoni, D., Mascherini, M. \& Manca, A. R. (2009). Scouting for drivers of the European knowledge society: the role of social participation. En M. D. Lytras, P. Ordoñez de Pablos, E. Damiani, D. Avison, A. Naeve \& D. G. Horner (Eds.), Best Practices for the Knowledge, Learning, Development, and Technology for All (pp. 319-327). Greece: Springer Berlin Heidelberg.

Wang, Q., Chen, X. \& Chen, Y. (2011). Development of a scale to measure residents' psychological empowerment in Chinese urban community. Journal of Community Psychology, 39(2), 202-211.

Wothke, W. (2010). Introduction to Structural Equation Modeling Course Notes. Carolina del Norte: SAS Institute Inc.

Recibido: 15 de abril, 2014 Aceptado: 4 de agosto, 2014 